\title{
The Evolutionary Origin of Cooperators and Defectors
}

\author{
Michael Doebeli, ${ }^{1 *}$ Christoph Hauert, ${ }^{1} \dagger$ Timothy Killingback ${ }^{2}$
}

Coexistence of cooperators and defectors is common in nature, yet the evolutionary origin of such social diversification is unclear. Many models have been studied on the basis of the assumption that benefits of cooperative acts only accrue to others. Here, we analyze the continuous snowdrift game, in which cooperative investments are costly but yield benefits to others as well as to the cooperator. Adaptive dynamics of investment levels often result in evolutionary diversification from initially uniform populations to a stable state in which cooperators making large investments coexist with defectors who invest very little. Thus, when individuals benefit from their own actions, large asymmetries in cooperative investments can evolve.

Cooperation is an enduring evolutionary conundrum (1-3). Its essence is captured by social dilemmas, in which groups of cooperators do better than groups of defectors, yet defecting individuals outcompete cooperative individuals in mixed groups. Considerable efforts have been expended trying to understand the evolution of cooperation on the basis of the prisoner's dilemma (3-9), which describes situations in which cooperative acts incur costs to the acting individuals, whereas benefits only accrue to others. In this case, cooperators are doomed in the absence of supporting mechanisms (3-9). In contrast, the social dilemma is relaxed if benefits of costly cooperative acts accrue not only to others but also to the cooperator itself. In classical game theory, this situation is described by the snowdrift game (also called hawk-dove game or chicken game), in which two drivers are trapped on either side of a snowdrift and have the options of staying in their cars or removing the snowdrift. Letting the opponent do all the work is the best option, but if both players refuse to shovel they can't get home. The essential feature of the snowdrift game is that defection is better than cooperation if the opponent cooperates but worse if the opponent defects. This maintains cooperation at a mixed stable state $(10-12)$.

Cooperative interactions in which benefits accrue to both donor and recipient are common (3). They arise when individuals in a population can produce a common resource that is accessible to everybody. For example, yeast produce an enzyme to hydrolyze

${ }^{1}$ Department of Zoology and Department of Mathematics, University of British Columbia, Vancouver, British Columbia V6T 1Z4, Canada. ${ }^{2}$ Department of Mathematics, College of William and Mary, Williamsburg, VA 23187, USA.

*To whom correspondence should be addressed. E-mail: doebeli@zoology.ubc.ca

$\dagger$ Present address: Program for Evolutionary Dynamics, Harvard University, One Brattle Square, Cambridge, MA 02138, USA. sucrose, and the enzyme secreted by a single individual can be used by the individual producing it as well as by others (13). It is natural to assume that cooperative investments, i.e., the time and effort spent in producing the common good, can vary continuously within a certain range and hence to extend classical games to evolutionary scenarios in which individuals can make continuously varying cooperative investments (14-16). Here, we define the continuous snowdrift game for the evolution of cooperative investments that incur costs to the donor and accrue benefits to both the donor and the recipient. Each individual has a continuous strategy, or trait, which we take to be a real number, $x$, between 0 and an upper limit, $x_{\max }$, and which represents the amount of investment that the individual makes in the cooperative enterprise. For simplicity, we assume that all interactions are pairwise, but the theory presented below immediately extends to interactions in groups of arbitrary size $N$ [Supporting Online Material (SOM) Text]. We define the payoff to an $x$ strategist interacting with a $y$ strategist to be $P(x, y)=B(x+y)-C(x)$, where $B(x+y)$ specifies the benefit that the $x$ strategist obtains from the total cooperative investment made by both agents and $C(x)$ specifies the cost incurred by the $x$ strategist due to its own investment. We assume that $B(x)$ and $C(x)$ are smooth, strictly increasing functions satisfying $B(0)=C(0)=0$.

In the continuous prisoner's dilemma (16), an $x$ strategist facing a $y$ strategist obtains the payoff $Q(x, y)=B(y)-C(x)$. Because in this case investments incur costs but no benefits to the investing player, investments always evolve to zero (16). In contrast, in the continuous snowdrift game one could expect that investments evolve away from zero to some intermediate level provided that benefits outweigh costs $[B(x)>C(x)]$ for small $x$. We will see, however, that the continuous snowdrift game displays much richer evolutionary dynamics.
We use adaptive dynamics (17-19) (SOM Text) to analyze the evolution of the strategy $x$. Consider a monomorphic population in which every individual adopts the same strategy, $x$. It follows from replicator dynamics (20) that the growth rate of a rare mutant strategy, $y$, in the resident $x$ population is $f_{x}(y)=P(y, x)-P(x, x)=B(x+y)-C(y)-$ $[B(2 x)-C(x)]$. The evolution of the trait $x$ is then governed by the selection gradient $D(x)=\partial f_{x} /\left.\partial y\right|_{y=x}=B^{\prime}(2 x)-C^{\prime}(x)$, and the adaptive dynamics of $x$ is described by $\dot{x}=$ $D(x)(17-19)$ (SOM Text).

Equilibrium points of the adaptive dynamics are called singular strategies and are solutions of $D\left(x^{*}\right)=B^{\prime}\left(2 x^{*}\right)-C\left(x^{*}\right)=0$. If there is no such solution, the trait $x$ montonically increases or decreases over evolutionary time, depending on the sign of $D(x)$. If $x^{*}$ exists, it is convergent stable and, hence an attractor for the adaptive dynamics, if $d D /\left.d x\right|_{x=x^{*}}=2 B^{\prime \prime}\left(2 x^{*}\right)-C^{\prime \prime}\left(x^{*}\right)<0(17-19)$ (SOM Text). If this inequality is reversed, $x^{*}$ is a repeller.

Initially, the population will converge to an attracting singular point $x^{*}$, but its subsequent evolutionary fate depends on whether $x^{*}$ is a maximum or minimum of the invasion fitness $f_{x}(y)$. If $x^{*}$ is a maximum, i.e., if $\partial^{2} f_{x^{*}} /\left.\partial y^{2}\right|_{y=x^{*}}=B^{\prime \prime}\left(2 x^{*}\right)-$ $C^{\prime \prime}\left(x^{*}\right)<0$, then $x^{*}$ is an evolutionary stable strategy (ESS), representing an evolutionary end state in which all individuals make intermediate cooperative investments. If, however, $B^{\prime \prime}\left(2 x^{*}\right)-C^{\prime \prime}\left(x^{*}\right)>0$, then a population of $x^{*}$ strategists can be invaded by mutant strategies on either side of $x^{*}$. In this case, the population undergoes evolutionary branching $(18,19)$ and splits into two distinct and diverging phenotypic clusters.

For example, such dynamics can be observed for quadratic cost and benefit functions $B(x)=b_{2} x^{2}+b_{1} x$ and $C(x)=c_{2} x^{2}+c_{1} x$. In this case, the singular point is unique if it exists and is given by $x^{*}=\frac{c_{1}-b_{1}}{4 b_{2}-2 c_{2}}$; it is an evolutionary branching point if $2 b_{2}<c_{2}<b_{2}<0$ (SOM Text). If evolutionary branching occurs, the two phenotypic clusters diverge and evolve to the borders of the trait interval (Fig. 1A) (SOM Text). The population emerging from this evolutionary process contains both defectors that do not make any investment at all and cooperators making the maximal investment (Fig. 1A). In this state, the continuous snowdrift game transforms into its traditional form with two coexisting strategies (SOM Text). Therefore, adaptive dynamics of continuous strategies yields a natural explanation of the evolutionary emergence of the pure cooperator and defector strategies of the traditional snowdrift game (21).

A complete classification of the adaptive dynamics for quadratic cost and benefit functions is provided in Fig. 1: evolutionary 

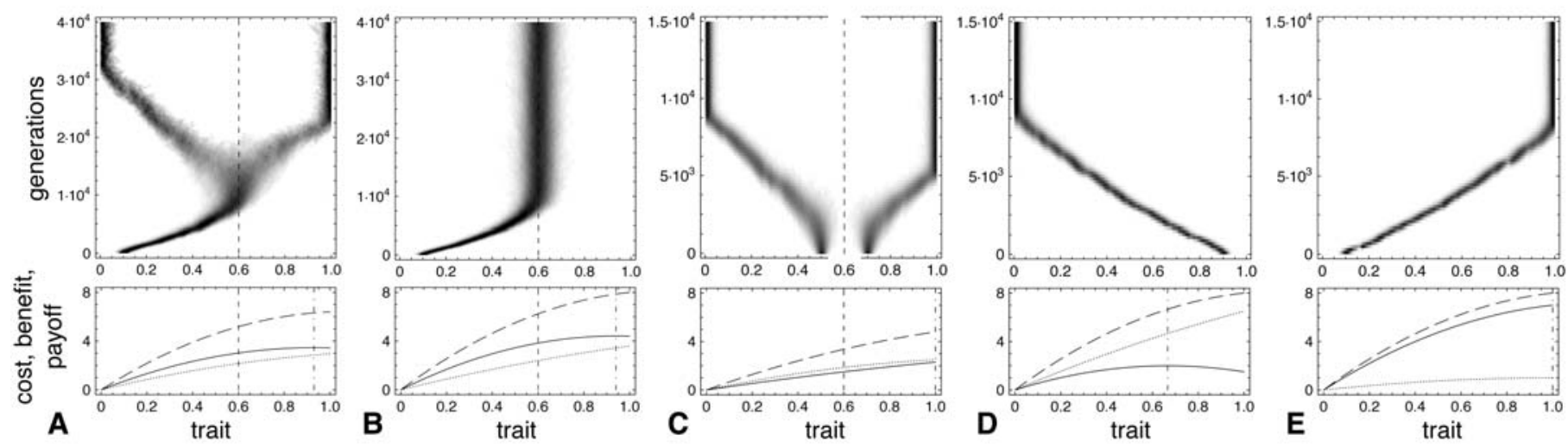

Fig. 1. Classification of evolutionary dynamics for quadratic cost and benefit functions $B(x)=b_{2} x^{2}+b_{1} x$ and $C(x)=c_{2} x^{2}+c_{1} x$. The top row shows the evolutionary dynamics of the trait distribution; darker shades indicate higher frequencies of a trait value. The singular strategies (dashed vertical lines) are indicated where appropriate. The bottom row shows the cost $C(x)$ (dotted line) and benefit $B(2 x)$ (dashed line) accrued in monomorphic populations, together with the mean payoff $B(2 x)-C(x)$ (solid line; the dash-dotted vertical line indicates maximal mean payoffs). (A) Evolutionary branching. (B) Evolutionarily stable singular strategy; note that population payoff is not maximized at the ESS. (C) Evolutionary repellor; depending on the initial conditions, the population either evolves to full defection or to full cooperation (two distinct simulations shown). ( $D$ and $E$ ) Unidirectional evolutionary dynamics in the

absence of singular strategies; in (D), cooperative investments decrease to zero, just as in the continuous prisoner's dilemma (16); in (E), full cooperation evolves. Results were obtained from numerical simulations of the continuous snowdrift game in finite populations (SOM Text). Parameter values were as follows: population size $N=10,000$, standard deviation of mutations $\sigma=0.005$, mutation rate $\mu=0.01$ (i.e., on average one mutation in the investment level per 100 offspring), and the following cost and benefit parameters: (A) $b_{2}=-1.4, b_{1}=6, c_{2}=-1.6, c_{1}=$ 4.56; (B) $b_{2}=-1.5, b_{1}=7, c_{2}=-1, c_{1}=4.6$; (C) $b_{2}=-0.5, b_{1}=3.4, c_{2}=-1.5$, $c_{1}=4.0$; (D) $b_{2}=-1.5, b_{1}=7, c_{2}=-1, c_{1}=8.0$; (E) $b_{2}=-1.5, b_{1}=7, c_{2}=$ $-1, c_{1}=2$. For all panels, the maximum investment was set to $x_{\max }=1$ and the parameters were chosen such that $B(x)$ and $C(x)$ are monotonically increasing functions on the interval $[0,1]$.

branching (Fig. 1A), convergent stable ESS (Fig. 1B), evolutionary repellor leading to bistable evolutionary dynamics (Fig. 1C), and, in the absence of a singular strategy, either uniform selection for defectors (Fig. 1D) or cooperators (Fig. 1E). We note that maximal mean payoffs in monomorphic populations cannot be used as a predictor of the evolutionary outcome (Fig. 1 and SOM Text). This is obvious in cases of evolutionary branching, but it is also generally true if the system exhibits an attracting ESS or unidirectional evolutionary dynamics (Fig. 1).

We illustrate the connection between the adaptive dynamics of the continuous snowdrift game and traditional game theory by considering local games between two strategies with similar investment levels (Fig. 2). For traditional two-player games, there are three basic types of outcomes (22): (i) one strategy dominates and reaches fixation, (ii) a globally stable mixed equilibrium occurs (as, for example, in the classical snowdrift game), or (iii) the game exhibits bistability, with one or the other strategy going to fixation depending on initial conditions.

Away from the vicinity of a singular point, the adaptive dynamics is directional, and the local game between a resident, $x$, and a nearby mutant, $x+\varepsilon(\varepsilon$ small, $>0)$, is of the first type, so that one of the two investment strategies is dominant. If an attracting singular point exists, successive local games exhibit dominance and induce evolutionary convergence to the vicinity of the singular strategy (Fig. 2A). Near the singular point, local games can be of any type. If the singular strategy is an ESS, local games between the singular strategy and neighboring strategies exhibit either domination by the ESS or bistability, and hence neighboring strategies cannot invade. In contrast, if the singular strategy is an evolutionary branching point, local games between the singular strategy and neighboring strategies always exhibit a mixed stable state; in particular, neighboring strategies can invade (Fig. 2A, insets). On the other hand, evolutionary repellors are characterized by the fact that local games on either side of the repellor again exhibit dominance but now induce directional evolution away from the singular point (Fig. 2B). This results in bistability of the global evolutionary dynamics.

More complicated evolutionary scenarios than those shown in Fig. 1 are possible with other cost and benefit functions. For example, the equation $B^{\prime}\left(2 x^{*}\right)-C^{\prime \prime}\left(x^{*}\right)=0$ may have more than one solution, in which case there are several singular points of the adaptive dynamics. Figure 3 shows two cases of an evolutionary branching point co-occurring with a repellor and illustrates that evolution does not always drive the phenotypic clusters emerging through evolutionary branching to the boundaries of the strategy space. Our numerical simulations (SOM Text) confirm that evolutionary branching is a generic and robust outcome for many different cost and benefit functions, $C(x)$ and $B(x)$, and always occurs under suitable conditions, i.e., whenever a singular point $x^{*}$ satisfies $2 B^{\prime \prime}\left(2 x^{*}\right)<$ $C^{\prime \prime}\left(x^{*}\right)<B^{\prime \prime}\left(2 x^{*}\right)<0$. These conditions require that both cost and benefit functions are saturating, i.e., have negative curvature, near the singular point. Saturating benefits are clearly realistic, whereas costs could often be expected to accelerate. However, diminishing additional costs of larger cooperative investments are reasonable whenever the initiation of cooperative acts, such as turning on enzyme production machinery, is more costly than subsequent increases in cooperative investments. Given an instance of evolutionary branching, it is in principle possible that further branching events occur that would lead to the establishment of more than two phenotypic clusters in the population $(18,19)$. In the case of quadratic cost and benefit functions, such secondary branching does not occur (SOM Text).

The paradox of altruism, of which the tragedy of the commons (23) is a celebrated avatar, is that although populations of altruists outperform populations of nonaltruists, selection will act to eliminate altruism altogether. Here, we have unveiled a different paradox of cooperation, which could be termed the "tragedy of the commune": In a cooperative system, in which every individual contributes to a common good and benefits from its own investment, selection does not always generate the evolution of uniform and intermediate investment levels but may instead lead to an asymmetric stable state, in which some individuals make high levels of cooperative investment and others invest little or nothing.

In practice, it is often difficult to determine the payoffs in social interactions and hence to distinguish prisoner's dilemma and 

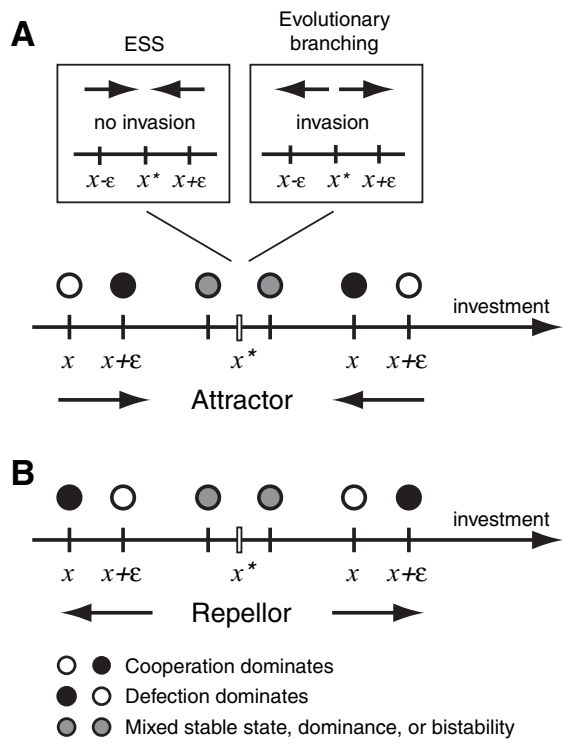

Fig. 2. Integrating traditional two-player games between similar investment strategies into global evolutionary dynamics. (A) Local games between investment strategies $x$ and $x+\varepsilon(\varepsilon$ small) induce convergence to an attracting singular point from below (cooperation dominates) and from above (defection dominates). Local games between strategies in the vicinity of the singular point can be of any type. Neighboring strategies can or cannot invade the singular strategy depending on whether the singular point is an evolutionary branching point or an ESS (insets). (B) If the singular point is a repellor, local games between investment strategies $x$ and $x$ $+\varepsilon$ ( $\varepsilon$ small) induce evolutionary divergence away from the repellor on either side.

snowdrift interactions [a phage system marks a rare exception, but interestingly, selection turns the prisoner's dilemma into a snowdrift game (24)]. Nevertheless, the mere existence of high- and low-investing individuals has often been taken as prima facie evidence that the interaction is governed by a prisoner's dilemma, with some additional mechanism, such as reciprocity, responsible for the coexistence of altruists and nonaltruists. The tragedy of the commune, however, provides a quite different and, in many ways, simpler explanation for the coexistence of high- and low-investing individuals, which potentially applies to a wide range of cooperative and communal enterprises in biological systems.

For example, in the aforementioned case of enzyme production in yeast, the tragedy of the commune suggests evolution toward a state in which some cells produce the enzyme whereas others do not and instead simply exploit the common resource. Exactly this situation has been found experimentally (13). The same mechanism may drive the evolution of defective interference in viruses (25). When viruses coinfect a cell, the replication enzymes they produce are a common resource. Selection could then favor diversifi-
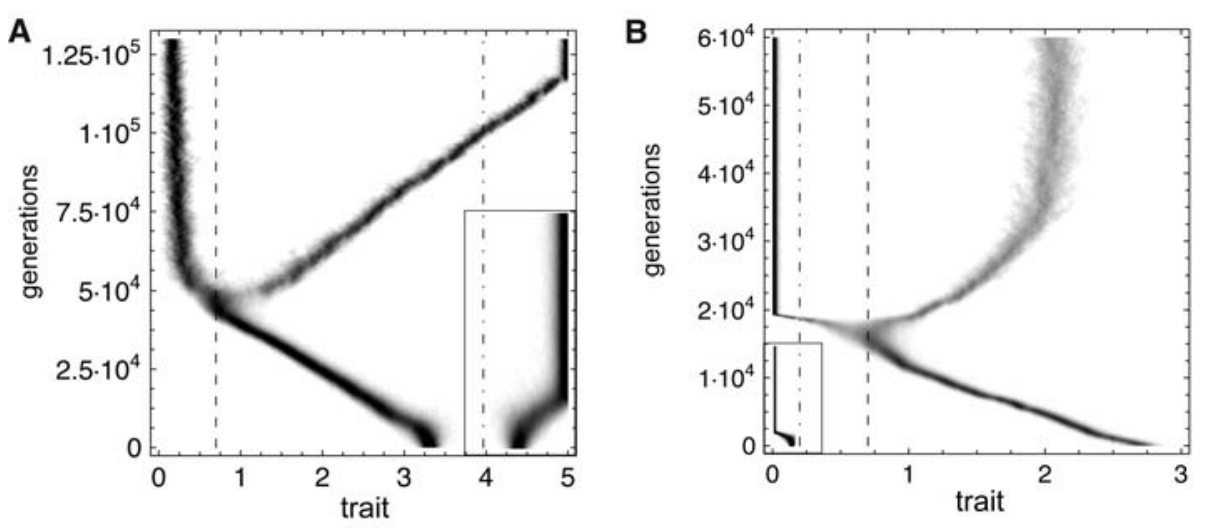

Fig. 3. Examples of simultaneous occurrences of a branching point (dashed line) and a repellor (dashdotted line) in the continuous snowdrift game. (A) $B(x)=b \sqrt{x}, C(x)=\ln (c x+1)(b=1$ and $c=0.6)$; if the population starts to the right of the repellor at $\hat{x} \approx 3.9$, cooperative investments continue to increase until the upper limit of the trait interval is reached (inset). However, if started below $\hat{x}$, the population evolves to the evolutionary branching point $x^{*} \approx 0.7$, where it splits into coexisting high-investing cooperators and low-investing defectors. In the defector branch investments do not evolve to zero, and due to the existence of the defector branch, the cooperator branch no longer "feels" the repellor. (B) $B(x)=b[1-\exp (-x)], C(x)=\ln (c x+1),(b=5$ and $c=10)$; the defective state is locally convergent stable (inset). Only populations that start out above the repellor $\hat{x} \approx 0.2$ evolve toward the branching point at $x^{*} \approx 0.7$ and subsequently split into cooperators and defectors. Cooperative investments saturate near $x^{*} \approx 2.1$, i.e., do not converge to the upper trait boundary, because of monotonically increasing costs and saturating benefits. Simulation parameters (SOM Text) were as follows: population size $N=10,000$, standard deviation of mutations $\sigma=0.005$, and mutation rate $\mu=0.01$.

cation into two coexisting viral types: one producing the replicase and the defective interfering particles in which replicase production is down-regulated (25). Similar remarks apply to social dilemmas in RNA phages due to coinfection of host cells (24). In all these examples, differences between cooperators and defectors have their basis in complex regulatory processes. Therefore, these differences likely involve multiple genetic changes, allowing for gradual evolution of different cooperative investment levels. We also note that one can draw interesting parallels between the tragedy of the commune and models for the evolution of anisogamy (i.e., for the emergence of asymmetric investments into gamete size $(26,27))$, which represents a common good in sexual species.

In behavioral ecology, classical examples of cooperation include collective hunting and territory defense in lions (28), predator inspection in sticklebacks (29), and alarm calls in meerkats (30). In theoretical discussions of these examples, the existence of cooperators providing a common good and defectors exploiting it has been assumed a priori. The tragedy of the commune, however, suggests an evolutionary mechanism for the emergence of distinct behavioral patterns with differing degrees of provisions to the common good. This mechanism may also apply to cultural evolution in human societies, in which large differences in cooperative contributions to communal enterprises could give rise to conflicts on the basis of accepted notions of fairness.

\section{References and Notes}

1. J. Maynard Smith, E. Szathmáry, The Major Transitions in Evolution (Freeman, Oxford, 1995).

2. S. A. Frank, Foundations of Social Evolution (Princeton Univ. Press, Princeton, NJ, 1998).

3. L. A. Dugatkin, Cooperation Among Animals: An Evolutionary Perspective (Oxford Univ. Press, Princeton, NJ, 1997).

4. R. Trivers, Q. Rev. Biol. 46, 35 (1971).

5. R. Axelrod, W. D. Hamilton, Science 211, 1390 (1981)

6. M. A. Nowak, R. M. May, Nature 359, 826 (1992).

7. M. A. Nowak, K. Sigmund, Nature 393, 573 (1998).

8. C. Hauert, S. De Monte, J. Hofbauer, K. Sigmund, Science 296, 1129 (2002).

9. E. Sober, D. S. Wilson, Unto Others: The Evolution and Psychology of Unselfish Behavior (Harvard Univ. Press, Cambridge, MA, 1998).

10. J. Maynard Smith, G. Price, Nature 246, 15 (1973).

11. R. Sugden, The Economics of Rights, Co-operation and Welfare (Blackwell, Oxford, 1986).

12. C. Hauert, M. Doebeli, Nature 428, 643 (2004).

13. D. Greig, M. Travisano, Biol. Lett. 217, S25 (2004).

14. M. Doebeli, N. Knowlton, Proc. Natl. Acad. Sci. U.S.A. 95, 8676 (1998).

15. L. M. Wahl, M. A. Nowak, J. Theor. Biol. 200, 307 (1999).

16. T. Killingback, M. Doebeli, Am. Nat. 160, 421 (2002)

17. U. Dieckmann, R. Law, J. Math. Biol. 34, 579 (1996)

18. S. A. H. Geritz, E. Kisdi, G. Meszéna, J. A. J. Metz, Evol. Ecol. 12, 35 (1998).

19. J. A. J. Metz, S. A. H. Geritz, G. Meszéna, F. J. A. Jacobs, J. S. van Heerwaarden, Stochastic and Spatial Structures of Dynamical Systems, S. J. van Strien, S. M. Verduyn Lunel, Eds. (North Holland, Amsterdam, 1996), pp. 183-231.

20. J. Hofbauer, K. Sigmund, Evolutionary Games and Population Dynamics (Cambridge Univ. Press, Cambridge, 1998).

21. Complementary interactive tutorials are provided at www.univie.ac.at/virtuallabs/ for further exploration of the evolutionary dynamics of the continuous snowdrift game.

22. M. A. Nowak, K. Sigmund, Science 303, 793 (2004).

23. G. Hardin, Science 162, 1243 (1968).

24. P. E. Turner, L. Chao, Am. Nat. 161, 497 (2003).

25. A. S. Huang, D. Baltimore, Comprehensive Virology, H. Fraenkel-Conrat, R. Wagner, Eds. (Plenum, New York, 1977), vol. 10, pp. 73-116. 
26. R. F. Hoekstra, J. Theor. Biol. 87, 785 (1980)

27. N. Maire, M. Ackermann, M. Doebeli, Selection 2, 119 (2001).

28. C. Packer, A. E. Pusey, Sci. Am. 276, 52 (1997).

29. T. Pitcher, Neth. J. Zool. 42, 371 (1993).

30. T. H. Clutton-Brock et al., Science 284, 1640 (1999).
31. The comments from one of the referees were particularly helpful. M.D. was supported by the Natural Sciences and Engineering Research Council (NSERC), Canada, and by the James S. McDonnell Foundation. C.H. was supported by the Swiss $\mathrm{Na}$ tional Science Foundation (SNF). The order of authors is alphabetical.
Supporting Online Material

www.sciencemag.org/cgi/content/full/306/5697/859/

DC1

SOM Text

References and Notes

15 June 2004; accepted 1 September 2004

\title{
A PINOID-Dependent Binary Switch in Apical-Basal PIN Polar Targeting Directs Auxin Efflux
}

\author{
Jiři Friml, ${ }^{1}$ Xiong Yang, ${ }^{2,3}$ Marta Michniewicz, ${ }^{1}$ Dolf Weijers, ${ }^{1,2}$ \\ Ab Quint, ${ }^{2}$ Olaf Tietz, ${ }^{4}$ René Benjamins, ${ }^{2,6}$ \\ Pieter B. F. Ouwerkerk, ${ }^{2}$ Karin Ljung, ${ }^{5}$ Göran Sandberg, ${ }^{5}$ \\ Paul J. J. Hooykaas, ${ }^{2}$ Klaus Palme, ${ }^{4}$ Remko Offringa ${ }^{2 *}$
}

\begin{abstract}
Polar transport-dependent local accumulation of auxin provides positional cues for multiple plant patterning processes. This directional auxin flow depends on the polar subcellular localization of the PIN auxin efflux regulators. Overexpression of the PINOID protein kinase induces a basal-toapical shift in PIN localization, resulting in the loss of auxin gradients and strong defects in embryo and seedling roots. Conversely, pid loss of function induces an apical-to-basal shift in PIN1 polar targeting at the inflorescence apex, accompanied by defective organogenesis. Our results show that a PINOID-dependent binary switch controls PIN polarity and mediates changes in auxin flow to create local gradients for patterning processes.
\end{abstract}

The plant signaling molecule auxin plays a central role in a wide variety of development processes. A major determinant in auxinmediated plant growth is the directed transport of auxin from foci of biosynthesis to sites of action. This polar auxin transport mediates vectorial gradients that underlie tropic growth responses and provide positional cues for apical-basal patterning, organogenesis, and vascular differentiation (1-4). The molecular characterization of the Arabidopsis thaliana pin-formed ( pin 1) mutant, which is defective in auxin transport and develops pin-like inflorescences, led to the identification of the PIN family of transporter-like membrane proteins. A substantial amount of data demonstrates that PIN proteins are important regulators of polar auxin transport that possibly function

${ }^{1}$ Developmental Genetics, Center for Molecular Biology of Plants, University Tübingen, Auf der Morgenstelle 3, D-72076 Tübingen, Germany. ${ }^{2}$ Developmental Genetics, Institute of Biology, Leiden University, Clusius Laboratory, Wassenaarseweg 64, 2333 AL Leiden, Netherlands. ${ }^{3}$ College of Life Sciences, Peking University, Beijing 100871, China. ${ }^{4}$ Albert-Ludwigs-Universität, Biologie II, Schaenzlestrasse 1, D-79104 Freiburg, Germany. ${ }^{5}$ Umeå Plant Science Center, Department of Forest and Plant Physiology, Swedish University of Agricultural Sciences, S 90183 Umeå, Sweden. ${ }^{6}$ Institute of Applied Genetics and Cell Biology, BOKUUniversity of Natural Resources and Applied Life Sciences, Muthgasse 8, A-1190 Vienna, Austria.

*To whom correspondence should be addressed. E-mail: offringa@rulbim.leidenuniv.nl as auxin efflux carriers (4). PIN proteins display asymmetric subcellular localization at the plasma membrane, which determines the direction of polar auxin transport and thus establishes the local auxin gradients that influence different developmental processes. The polarity of PIN proteins can be rapidly modulated in response to external or developmental cues $(1,3,5)$, a process that is enabled by continuous GNOM ARF GEF-dependent cycling of PINs between endosomes and the plasma membrane (6) (GNOM, Arabidopsis GNOM protein; ARF, ADP ribosylation factor; GEF, guanine nucleotide exchange factor).

Loss-of-function mutants of the protein serine-threonine kinase PINOID (PID) display apical organogenesis defects similar to those of the pin1 mutant (7). Constitutive overexpression of PID (35S::PID), but not of the kinase-negative MPID (35S::MPID), leads to hypocotyl and root agravitropy and to loss of the primary root meristem function $(8,9)$. The collapse of the root meristem in $35 S: \because P I D$ seedlings, which is characterized by the loss of meristem initials followed by terminal differentiation, is restricted to the primary root and is preceded by a reduction in auxin-responsive DR5::GUS expression (Fig. 1, A and B) (9). Measurements of indole-3-acetic acid (IAA) in intact root tips showed that IAA levels are significantly reduced in $35 S:: P I D$ primary root tips as compared to wild-type root tips (Fig. 1E). In contrast, free IAA concentrations in lateral root tips of 8- to 11-day-old $35 S: \because P I D$ and wild-type seedlings do not differ significantly (Fig. 1E), and accordingly the DR5::GUS expression peak is unchanged (Fig. 1, C and D). These data confirmed that PID overexpression results in reduced auxin accumulation in the primary root tip, thereby causing a reduction in the DR5 expression peak in the root meristem and eventually the collapse of this structure. Treatment with the auxin efflux inhibitor naphthylphtalamic acid (NPA) restores the DR5 expression peak and prevents root meristem collapse (9), whereas treatment with auxin itself has no effect. These data suggest that PID is a regulator of NPA-sensitive polar auxin transport.

We used the timing of root collapse as an assay to address whether PID action on auxin transport occurs through PIN efflux regulators. $35 S: \because P I D$ plants were crossed with lossof-function mutants of PIN genes known to mediate root development, these being PIN2, PIN3, and PIN4 $(5,10,11)$. In the pin2/eirl-1 and pin4 mutant backgrounds, the $35 S:: P I D$ mediated root collapse was significantly delayed, whereas the pin 3 mutation resulted in a mild delay in root collapse around 4 to 5 days after germination (Fig. 1F). Both pin2 and pin4 mutations result in increased auxin concentrations in the root: pin 2 elevates auxin concentrations because of the lack of redistribution of auxin via basipetal transport from the root tip to the elongation zone (12), and pin4-elevated auxin levels result from the absence of a focused PIN4-driven auxin sink in the first columella tier (11).

These results imply that PID gain of function changes auxin concentrations in the root tip through the PIN proteins. Conceivably, PID could regulate either the expression of PIN proteins, the polarity of their subcellular localization, or their activity. Because an activity assay for PIN proteins is so far not available, we focused on testing the expression and subcellular localization of PIN proteins, by immunolocalizing various PIN proteins in primary and lateral root tips of wild-type and $35 S: \because P I D$ lines. This showed that the tissue-specific expression domains of the PIN proteins are unchanged in $35 S: \because P I D$ root tips. Although quantitative changes cannot be excluded, this suggests that regulation of PIN gene expression or protein stability is not a primary target of PID action. 35S::PID expression did, however, lead to a basalto-apical shift in the subcellular polarity of PIN proteins. This apical shift was most ap- 


\title{
Supporting Online Material:
}

\section{Adaptive Dynamics of Continuous Games}

\author{
Michael Doebeli ${ }^{1}$, Christoph Hauert ${ }^{1,2}$ \& Timothy Killingback ${ }^{3}$ \\ ${ }^{1}$ Departments of Mathematics and Zoology, University of British Columbia, Vancouver BC V6T 1Z4, \\ Canada. \\ ${ }^{2}$ Present address: Program for Evolutionary Dynamics, Harvard University, One Brattle Square, Cam- \\ bridge MA 02138, USA. \\ ${ }^{3}$ Ecology and Evolution, ETH Zürich, 8092 Zürich, Switzerland.
}

\section{Adaptive Dynamics}

The evolution of a continuous trait $x$ under mutation and selection can be analyzed using the mathematical framework of adaptive dynamics $(S 1, S 2, S 3)$. The central concept is that of invasion fitness $f_{x}(y)$, which denotes the growth rate of a rare mutant $y$ in a resident population that is monomorphic for trait $x$. The adaptive dynamics of the trait $x$ is then governed by the selection gradient $D(x)=\partial f_{x} /\left.\partial y\right|_{y=x}$, so that $\dot{x}=D(x)$. For a detailed discussion of the underlying assumptions of this dynamic equation we refer to $(S 1, S 2, S 3)$. Note that in general, $\dot{x}=m D(x)$, where $m$ depends on population size and reflects the mutational process providing the raw material for evolutionary change. For constant population sizes, $m$ is simply a parameter that scales time, and one can set $m=1$ without loss of generality.

Singular points of the adaptive dynamics are given by solutions of $D\left(x^{*}\right)=0$. If there is no such solution, the trait $x$ either always increases or decreases evolutionarily, depending on the sign of $D(x)$. If a singular trait $x^{*}$ exists, it is convergent stable - and hence an attractor for the adaptive dynamics - if $d D /\left.d x\right|_{x=x^{*}}<0$. If this inequality is reversed, $x^{*}$ is a repellor, i.e. the trait $x$ evolves to ever lower values if the initial trait of the population $x_{0}$ is $x_{0}<x^{*}$, and to ever higher values if $x_{0}>x^{*}$.

Very interesting evolutionary dynamics can occur because convergence stability does not imply evolutionary stability. Generically, a convergent stable singular point $x^{*}$ is either a 
maximum or a minimum of the invasion fitness $f_{x}(y)(S 2, S 3)$. If $x^{*}$ is a maximum, i.e., if $\partial^{2} f_{x^{*}} /\left.\partial y^{2}\right|_{y=x^{*}}<0$, then $x^{*}$ is evolutionarily stable, i.e., it cannot be invaded by any mutant. If, however, $x^{*}$ is a minimum, i.e., if $\partial^{2} f_{x^{*}} /\left.\partial y^{2}\right|_{y=x^{*}}>0$, then a population that is monomorphic for $x^{*}$ can be invaded by mutants with trait values on either side of $x^{*}$. In this case, the population first converges evolutonarily towards $x^{*}$, but subsequently splits into two distinct and diverging phenotypic clusters. This phenomenon is called evolutionary branching, and the singular point is called an evolutionary branching point.

\subsection{Continuous Snowdrift Game}

In the Continuous Snowdrift game, the quantitative trait $x$ represents the level of cooperative investments. The growth rate of a rare mutant strategy $y$ in a monomorphic resident $x$ is determined by replicator dynamics $(S 4)$, and the invasion fitness of the rare mutant $y$ is given by $f_{x}(y)=P(y, x)-P(x, x)$, where $P(y, x)$ is the payoff of $y$ playing against $x$, and $P(x, x)$ is

the payoff of $x$ playing against itself. In the Continuous Snowdrift game the payoff is given by $P(x, y)=B(x+y)-C(x)$ where $B(x)$ and $C(x)$ are the benefit and cost functions.

The adaptive dynamics of the continuous trait $x$ is then given by $\dot{x}=D(x)=B^{\prime}(2 x)-$ $C^{\prime}(x)$, and singular strategies are solutions of $B^{\prime}\left(2 x^{*}\right)=C^{\prime}\left(x^{*}\right)$. If a singular strategy $x^{*}$ exists, it is convergent stable if $d D /\left.d x\right|_{x=x^{*}}=2 B^{\prime \prime}\left(2 x^{*}\right)-C^{\prime \prime}\left(x^{*}\right)<0$, and it is evolutionarily unstable if $\partial^{2} f_{x^{*}} /\left.\partial y^{2}\right|_{y=x^{*}}=B^{\prime \prime}\left(2 x^{*}\right)-C^{\prime \prime}\left(x^{*}\right)>0$. Consequently, $x^{*}$ is a branching point if $2 B^{\prime \prime}\left(2 x^{*}\right)<C^{\prime \prime}\left(x^{*}\right)<B^{\prime \prime}\left(2 x^{*}\right)<0$. Note that adaptive dynamics never maximizes the monomorphic population payoff given by $B(2 x)-C(x)$, because maximizing this payoff would yield the gradient dynamics $2 B^{\prime}(2 x)-C^{\prime}(x)$, which is different form the adaptive dynamics given above.

\subsubsection{Quadratic Cost and Benefit Functions}

If the cost and benefit functions $B(x)$ and $C(x)$ are linear, the gradient $D(x)$ is constant and the evolution of the trait $x$ is always directional, either leading to ever higher or ever lower investments (except in the degenerate case where $x$ is a neutral trait). However, richer dynamics are already observed for quadratic cost and benefit functions $C(x)=c_{2} x^{2}+c_{1} x$ and $B(x)=$ 
$b_{2} x^{2}+b_{1} x$, for which the adaptive dynamics are given by $\dot{x}=4 b_{2} x+b_{1}-2 c_{2} x-c_{1}$. The singular strategy (if it exists) is given by $x^{*}=\frac{c_{1}-b_{1}}{2\left(2 b_{2}-c_{2}\right)}$ and is convergent stable if $2 b_{2}-c_{2}<0$ and evolutionarily stable if $b_{2}-c_{2}<0$.

The existence of a positive $x^{*}$ requires either $(i) 4 b_{2}-2 c_{2}>c_{1}-b_{1}>0$ or $(i i) 4 b_{2}-2 c_{2}<$ $c_{1}-b_{1}<0$. In the first case, $x^{*}$ is always a repellor. Whether the repellor is evolutionarily stable or not is irrelevant as this state is never reached from generic intitial conditions. In the second case $x^{*}$ is always convergent stable. If, in addition, $b_{2}-c_{2}<0$, then $x^{*}$ is evolutionarily stable strategy (ESS). In this case, the population evolves towards the evolutionary end state $x^{*}$ in which all individuals make intermediate cooperative investments. If, however, $b_{2}-c_{2}>0$, then $x^{*}$ is an evolutionary branching point, and after converging towards $x^{*}$, the population splits into two distinct and diverging phenotypic clusters.

\subsubsection{Adaptive Dynamics after Branching}

After evolutionary branching, the adaptive dynamics can be calculated based on the equilibrium frequencies of the two co-existing strategies $x>x^{*}>y$ (where $x^{*}$ is the branching point). According to traditional replicator dynamics (S4) the equilibrium frequency $p^{*}$ of strategy $x$ is the solution of $p^{*} P(x, x)+\left(1-p^{*}\right) P(x, y)=p^{*} P(y, x)+\left(1-p^{*}\right) P(y, y)$. With quadratic cost and benefit functions, this yields $p^{*}=\frac{c_{1}-b_{1}+x\left(c_{2}-b_{2}\right)+y\left(c_{2}-3 b_{2}\right)}{2 b_{2}(x-y)}$. The invasion fitness of a mutant $v$ with respect to the two resident branches $x$ and $y$ is then given by $f_{x, y}(v)=p^{*} P(v, x)+(1-$ $\left.p^{*}\right) P(v, y)-\bar{P}(x, y)$ where $\bar{P}(x, y)=p^{*} P(x, x)+\left(1-p^{*}\right) P(x, y)=p^{*} P(y, x)+\left(1-p^{*}\right) P(y, y)$ denotes the average population payoff. Adaptive dynamics in the two branches is then given by $\dot{x}=\left.m_{1}(x, y) \frac{\partial f_{x, y}(v)}{v}\right|_{v=x}$ and $\dot{y}=\left.m_{2}(x, y) \frac{\partial f_{x, y}(v)}{v}\right|_{v=y}$, respectively, where $m_{1}(x, y) \propto p^{*}$ and $m_{1}(x, y) \propto 1-p^{*}$ are positive quantities describing the mutational process in the two branches $(S 2, S 3)$. For the quadratic cost and benefit functions one finds $\dot{x}=m_{1}(x, y)\left(b_{2}-c_{2}\right)(x-y)$ and $\dot{y}=-m_{2}(x, y)\left(b_{2}-c_{2}\right)(x-y)$. This implies that after branching, evolution is always directional and drives the trait values in the two branches to the boundaries of the strategy range. With more complicated cost and benefit functions, such as those used for Fig. 3 (see main text), analytical solutions for $p^{*}$, and hence for the 2-dimensional adaptive dynamics, can in general not be obtained. 


\subsubsection{Groups of $N$ Interacting Individuals}

The Continuous Snowdrift can be generalized to groups of $N>2$ interacting individuals, in which the $N$ individuals make cooperative investments $x_{1}, \ldots, x_{N}$ towards a common good. There are a number of ways in which this can be done, but perhaps the most straightforward approach is to assume that the payoff to the $i$-th player is $\frac{1}{N} B\left(\sum_{j=1}^{N} x_{j}\right)-C\left(x_{i}\right)$, reflecting the fact that the benefit accrued from the sum of the individual investments is equally shared among the $N$ interacting players. In principle, the factor $1 / N$ can be incorporated into the benefit function, but explicitly retaining it facilitates comparison of results for different group sizes $N$ (see below). The payoff of a mutant $y$ in a monomorphic resident population with strategy $x$ is $P(y, x)=\frac{1}{N} B((N-1) x+y)-C(y)$. Using similar arguments as above, this yields the adaptive dynamics $\dot{x}=\frac{1}{N} B^{\prime}(N x)-C^{\prime}(x)$. Singular strategies $x^{*}$ are now given as solutions of $\frac{1}{N} B^{\prime}\left(N x^{*}\right)-C^{\prime}\left(x^{*}\right)=0$. A singular strategy is convergent stable if $B^{\prime \prime}\left(N x^{*}\right)-C^{\prime \prime}\left(x^{*}\right)<0$, and it is evolutionarily stable if $\frac{1}{N} B^{\prime \prime}\left(N x^{*}\right)-C^{\prime \prime}\left(x^{*}\right)<0$. The condition for evolutionary branching becomes $B^{\prime \prime}\left(N x^{*}\right)<C^{\prime \prime}\left(x^{*}\right)<\frac{1}{N} B^{\prime \prime}\left(N x^{*}\right)<0$.

With quadratic cost and benefit functions, the singular strategy for $N$-player games is thus given by $x^{*}=\frac{c_{1}-\frac{1}{N} b_{1}}{2\left(b_{2}-c_{2}\right)}$. In complete analogy to pairwise interactions, the existence of $x^{*}$ requires either (i) $2\left(b_{2}-c_{2}\right)>c_{1}-\frac{1}{N} b_{1}>0$ or (ii) $2\left(b_{2}-c_{2}\right)<c_{1}-\frac{1}{N} b_{1}<0$. In the first case, $x^{*}$ is again a repellor. In the second case it is an ESS if $b_{2}-c_{2}<0$, while $x^{*}$ is an evolutionary branching point if $b_{2}<c_{2}<\frac{1}{N} b_{2}<0$. It follows that the range of parameters generating evolutionary branching increases with group size $N$.

\section{Individual-Based Simulations}

The analytical predictions derived from adaptive dynamics can be illustrated and verified through individual-based simulations of the Continuous Snowdrift game in finite populations of fixed size $N_{\text {pop }}(S 5)$. These simulations emulate replicator dynamics in populations in which individuals are characterized by their investment strategy $x$. The population is updated asynchronously by sequentially choosing a random focal individual $x$ to be replaced by an offspring as follows. The payoff of the focal individual, $P_{x}=P(x, z)$, is determined through a single interaction 
with a random member $z$ of the population. $P_{x}$ is then compared to the payoff of another randomly chosen individual $y$, whose payoff $P_{y}$ is also obtained through a random interaction. With a probability $w$ proportional to the payoff difference, $w=\left(P_{y}-P_{x}\right) / \alpha$ (where $\alpha=\max _{x, y, u, v}|P(x, y)-P(u, v)|$ ensures $\left.w \leq 1\right)$, the offspring replacing the focal individual has $y$ as parent; otherwise the parent is the focal individual itself (which is also the case if $P_{y}<P_{x}$ ). The offspring inherits the parental strategy, except if a mutation occurs (which happens with probability $\mu$ ), in which case the offspring strategy is drawn from a Gaussian distribution with the parental strategy as mean and a small standard deviation $\sigma$. This numerical scheme implements the deterministic replicator dynamics in the limit of large population sizes $N_{\text {pop }}$ (S6). Our individual-based models of the Continuous Snowdrift game can be explored interactively at http://www.univie.ac.at/virtuallabs.

\section{References and Notes}

S1. U. Dieckmann, R. Law, J. Math. Biol. 34, 579 (1996).

S2. S. A. H. Geritz, E. Kisdi, G. Meszena, J. A. J. Metz, Evol. Ecol. 12, 35 (1998).

S3. J. A. J. Metz, S. A. H. Geritz, G. Meszena, F. J. A. Jacobs, J. S. van Heerwaarden, Stochastic and Spatial Structures of Dynamical Systems, S. J. van Strien, S. M. Verduyn Lunel, eds. (North Holland, Amsterdam, 1996), pp. 183-231.

S4. J. Hofbauer, K. Sigmund, Evolutionary Games and Population Dynamics (Cambridge University Press, Cambridge, 1998).

S5. C. Hauert, Virtuallabs: Interactive tutorials on evolutionary game theory, http://www.univie.ac.at/virtuallabs (2004).

S6. C. Hauert, M. Doebeli, Nature 428, 643 (2004). 